apparell en bon itat, a condut la plupart des exploitants à adopter smmullanement plusieurs systèmes de déchargents montés en parallele, an moms dans les postes mporlants.

Si l'on emplore plusieurs déchargeurs à rornes, il faudra leur downer un réglage différenl, de maniòre quils ne fonchonnent que successivement.

Pour chacun des types de dichargenrs, on ardoptera de préfét rence, pour la protection d'une ligne polyphasée, lo montage tht mulipte. Co montage est tel quil rxiste deux dichargenrs en sérue entre les phases prises deux à deux, el deux déchargeurs également en série entre chaque phase et la terre. Nous avons vu notamment que ce montage avart cté recomnu indispensable pour la muse du nentre it la terre.

Quelques eviplollanls conservent des fusibles en série sur les dlikhal geurs, han ulue ce système al, l'moonvénient de supprimer lapparell chaque fois quil a fonclionné, ce dont on peut me pas s'apercevon de sulte.

Enfin, dans les installations au-lessus de 5000 volls, il convent do renfermer les díchargeurs entrc des clousons isolantes.

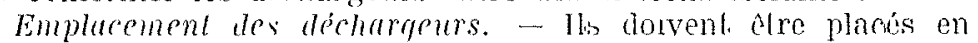

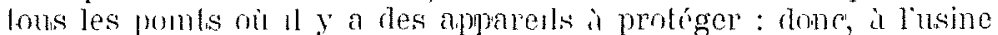
rui sera placé le poste le plus complet, et aux sous-stahons; dans les lignes mixtes, an raccordement, des cables avec les lignes aériennes.

De plus, dans les transmissions el distributions aériennes, surtoni en pays de montagne, ll existe des palıes de ligne plus fréfuemmenl, exposíes aux orages. Il est dlune pralique courante d'y placor des postes do dérhargeus, mass ceux-ce selont peu fa-

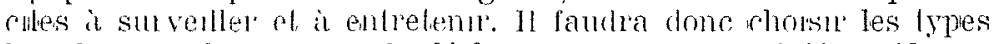
les plus smmples, comme le dlschargeur à colnes, róglés suffisamment haut pour iviter les fonctuonnements intempestufs par sute de chute de con ps intranger's dans l'intervalle.

11I. Protection Générale des Appareils. - Malgré toutes les précaulıons prises, des surtensions peuvent avoir passé devant les déchargeurs, el parvenır jusqu'aux appareıls..

be plus, conme Potier lavat fat observer, une onds de sur. tension, msulfisanle pour rompre l'isolant des lignes, peul se propager ave un tront lrès raide, en sorte que deux points de la ligne, bien que trìs rapprochés, peuvent se trouver portés ì $\mathrm{mm}$ polentiel trins dilfinenl. Si ces deux pomts sont les fils de deux sptres virsines d'm entoulement, et que l'isolant de ces fils n'ait pas até phévu assez forl, cet isolant sera percé. Il est done nécessarre de prentre des dispositions pour allonger le front d'onde.

Les bobmes de réaction 'servent pour ces deux cas. Elles sont conshturcs quelinuefois par le fil de ligne lu1-meme, ou par une lame curoulie en spuale el très soigneusement solée. Celte prabque est, croyoms nous, unversellemenl enrployce pour la prolction des apparesls. Jille est, en tous cas, d'ume applicalion plus facile et moms conteuse que le ienforcement de l'solant, vers l'extrémitó des enroulements, préconsé par certams ingémeurs américains.

Il faudra, bien entendu, mstaller cotle protection non seulement à l'entréc de l'usine, mass anisst ì l'entrée de tous les postes de transformateurs of an racordement avee les parties sonterrames.

contrôle du fonctionnement des dichargeurs. - Co comlroble est de phas souvelly sommane, of pour emse, puisqu'on ne pent guetter indífinment, le fonctionncment d'm déchargeur.

Les Amórıcains préconsent beaucoup l'emplos de papiers témoins, placís par exemple dans l'un des intervalles d'un déchargenr i rylindre ou lans licclatemr ì cornes. Le papter, ignifugé, est trour d'me manicre différente sulvant l'énergie véhiculce par la déchange. Les Trunsuctions of The American Instutute of Electrical Engineers ont reprodul des photographess intéressintcs de res papters. Mas il fanl nolor qu'sl penl passer plus d'une décharge par lo méme tron avant que le japier ait sté remplacé, en sorle gura, le plas souverul, on ne relèvo que la jésultante de plusieurs décharges.

Gonclusions.. - Les conclusions de ce traval pourront etre que la question des déchargems, condamnce, par son essence mème, ì rester dans le domano purement expérunental, n'a pas été suffisamment étudı́e jusqu'ıcı. On veut des appareils protégeant l'installation sans que des perturbalions puissent résulter de lenr fonctionnement.

Comme, rh definhtuve, res apparesls sont pen conteux, on se contente a'approximatıon, quile a multipher les postes. Il serait interessant copendant que quelques exploitants de lows veullent bıen se rlévouer à préciser par l’expúrıence quelques règles générales qui épargneraient à leurs collègues des dépenses inutiles rie temps et d'argent.

\section{USINE HYDRO-ÉLECTRIQUE DE SÉCHILIENNE}

Cette usine est située sur la Romanche, au lieu dit Pontde-Gavet, à 600 mètres environ en amont de la station de Séchilienne du tramway de Grenoble à Bourg-d'Oisans. Elle fonctionne en parallèle avec l'usine d'Avignonnet, sur le Drac, pour alimenter lo vaste réseau de la Sociéte Greno. bloise de Force et Lumière.

La Romanche, bien connue des alpinistes, prend sa source aux glacier's dauphinois de la Meije et dı Pelvoux, Son débit varie de 8 à $10 \mathrm{~m}^{3}$ en étiagse,à $200 \mathrm{~m}^{3}$ en clues,avec une moyellue annuelle de $30 \mathrm{~m}^{3}$ environ. Par suite des variations de températures diurnes et nocturnes qui activent ou ralentissent la fonte des neiges et glaces de la haute montagne, le clébit oscille périodiquement suivant les heures d'une mòme journéo : La Romanche monte de 6 heures du sor à minuit, ce qui montre que l'eau met à peu près 8 heures pour descendro des glacicrs jusqu'à Séchilienne (60 à 70 kms), le maximum de la fonte ayant sensiblement lieu de 10 heures du matin à 4 heuros du soir, et que le temps do la montce est heaucoup plus court que celui de la descente.

Aménagement hydraulique. - Cet aménagement a nécessité l'établissement d'un bairrage mobile de prisc d'eau, d'une chambre de décantation, d'un canal d'amenée en tunnel, d'une chambre de mise en charge également en tunnel, et d'une conduite forcée.

Barrage. - Le barrage de prise d'eau est établi un peu au-dessus du village de Gavet. Il est constitué par leux grandes vannes métalliques, de $12 \mathrm{~m}$. d'ouverture et de $3 \mathrm{~m}$. de hauteur, s'appuyant d'uno part sur des piliers latéraux établis de chaque côté de la rivière, et d'autre part sur un pilier central ayant $8 \mathrm{~m} 60$ de hauteur, $7 \mathrm{~m}$. de longueur et de $2 " 150$ de largeur.

La poussée normale qui s'exerce sur chaque vanne, lorsque celle-ci est levée de manière que le niveau de l'cau amont affleure sa crête, est de 54 tonnes. Mais cette poussée peut être dépassée si l'eauvient à surpasser sa crête. Aussi, pour pouvoir manceuvrer facilement et rapidement les vannes, était-il indispensable d'interposer entre elles et leurs supports des galets substituant le roulement au glissement.

En outre, chaque vanne est en partie équilibre par un système de contropoids représenté par la fiøure 1 . A 1 m50 de chaque extrémité, on a disposé une poulie $P$, fixée à la
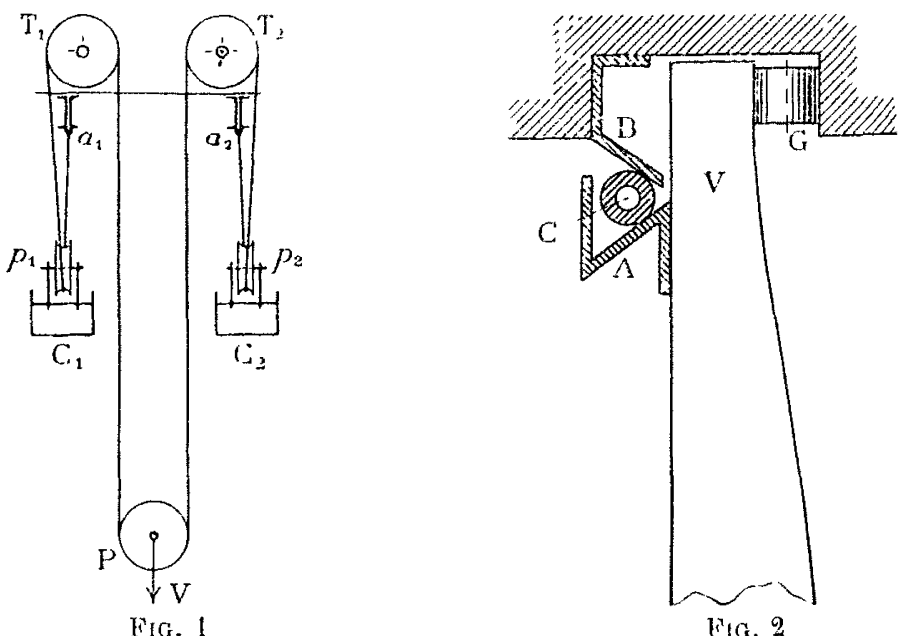

Fıgures schématiques montrant les dispositı́s d'équilıbrage et d'étanchérté d's vannes du barrage de prise d'eau.

vanne $V$, sous laquelle passe un câble qui s'enroule sur deux treuils $T$ installés sur une passerelle superrıcure. A l'amont et a l'aval de la vanne, un contrepoids $\mathrm{C}$, constitué par un caisson rempli de pierres, est suspendu à une poulie $p$ autour de laquelle est enroulé un 
câble dont une extrémité est fixée à la passerelle en $\iota$, et dont l'autre extrémité s'enroule sur les treuils 'T. Ce disposilif réduit la hauteur du déplacement vertical des contrepoids C, qui n'est égal qu'à celui de la vanne.

Les vannes sont constituées par une charpente métallique composée de 5 poutres horizontales. ayant chacune la forme d'un solide d'égale résistance, et réunies à leur's extrémités par des pièces verticales qui servent en même temps de chcmin de roulement pour les galets $\mathrm{G}$; ces poutres sont toutes semblables, elles sont seulement de plus en plus rapprochées au fur et ì mesure que la profondeur s'accroit, de manicre a les faire tlavailler toutes au même taux.

L'ćtanchéité est obtenue au moyen d'un dispositil représenté par la figure 2 . Un fer on $Z, A$, ('st fixé à chacune des extrémités amont sur toute la hauteur de la vanne $\mathrm{V}$; une cornière $\mathrm{B}$ esl fixce à la paroi du pilier. lintre ces deux fers ost intercalée uno barre ronde $\mathrm{C}$ que la pression de l'eau applique continuellement contre Jes fors. Lor'squ'on manoeuvre la vanne, on n’a an $=\mathrm{i}$, comme surface frotlante soumise à la pression de l'eau, que la surface du plan vertical limité entre les génératrices de contact de C sur A et B.

Sur la rive gauche, en dehors du pilier, on a méllagé une échelle a poisson.

Prise decu. - La prise d'eau s'effectue sur la rive droite de la Romanche, au moyen d'un canal I-II qui s'ouvre sur la rivière immédiatement en avant des vannes. L'eau pénètre dans ce canal en passant par dessus un mur $a b$ dont la crête est arrasée a $1^{m 5} 50$ en controbas du nivcau normal de la retenue créée par les vannes. Grace à ce dispositif, les cailloux et graviers,

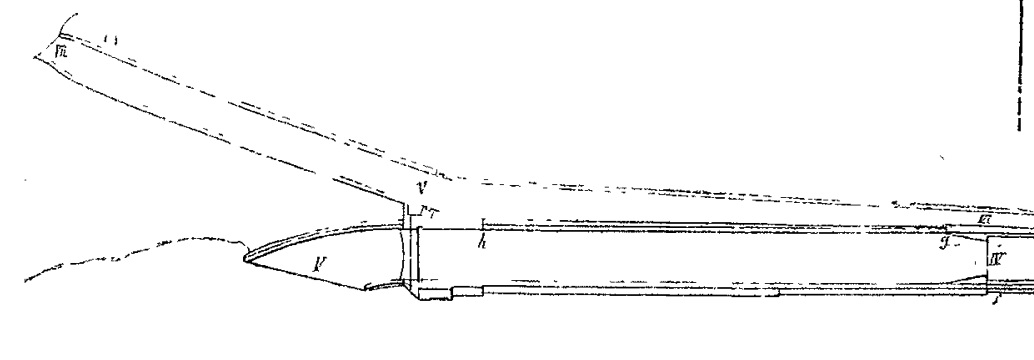

chroriés par la Romanche en temps de crue, glissent „e long de ce mur sans pouvoir pénétrer dans le canal. Ainsi que le montre la figure 3 , co canel de prise d'eau contourne le pilier extérieur de la vanne rive droite et retourne à la Romanche. Il est termmé par une vanne, fermee en basses eaux, (fui permet d'évacuer les gros sables qui s'y sonl déposé-i. Cette vanne est visible sur la fig. 4, ou alle masque en partie la vanno de droite du barlage de prise d'eau.

l'eau amenée par ce premier canal pénètre dans un second canal III-IV, on passant par dessus un mur $c d$ dont la crète cst arraséc à $1 \mathrm{~m}$. en contrebas du niveau normal. La profondeur de ce canal est de $2 \mathrm{~m}$. et sa lar. geur de $4 \mathrm{~m}$. Le mur qui le sépare de la rivière forme dévorsoir sur $65 \mathrm{~m}$. de longueur, de $e$ en $f$. A partir du point $f$, la profondeur est Jrusquement augmentée, et portée d $5 \mathrm{~m}$, afin d'augmenter la section transversale et d'obtenir uno vitesse de l'eau asscz faiblo pour permettre le dépôt des menus sables. Cette partic IV-V du canal constitue la chambre de décantation. Elle se termine pal des vannes ảe fond, permettant d'évacuel les dépìts.

Sur la droite de cette chambre de decantation, et sur toute sa longueur ( $55 \mathrm{~m}$.), se trouvent les grilles $g h$, qui ont $2 \mathrm{~m}$. de profondeur, of reposent sur un mur de $3 \mathrm{in}$. de hauteur. Elles sont constituées par des fer's méplats, espa- cés de $20 \mathrm{~mm}$, ct groupés en panneaux interchangeables par groupes de 10 barreaux. L'eau qui traverse ces grilles pénètre dans une seconde chambre VI-VII, qui a $4 \mathrm{~m}$

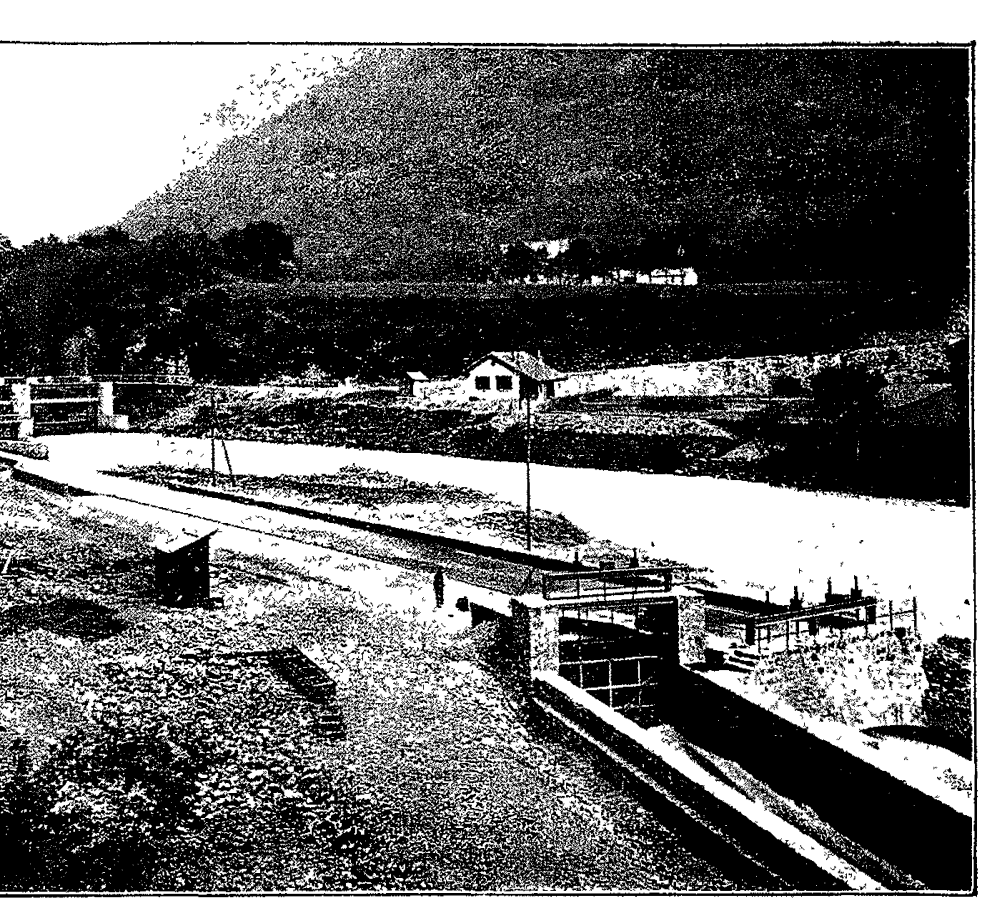

Fig. f. - Vue genérate de la prise dieau.

de largeur et $2 \mathrm{~m}$. de profondeur, et qui constitue la tête du canal d'amence proprement dit; en face des vannes de chasse de la chambre de décantation se trouve une vanne d'arrêt $\mathrm{V}$ qui permet d'isoler le canal d'amenée de la prise d'eau en cas de réparation. A l'autre extrémité de cette chambre VI-VII, en amont, se trouve une vanue de secours IV qui permet dintroduire l'eau directement sans passer par les grilles, au cas où celles-ci viendraient à être obstruées par la gelée.

Fig. 3. - Vlan de la prise d eau.

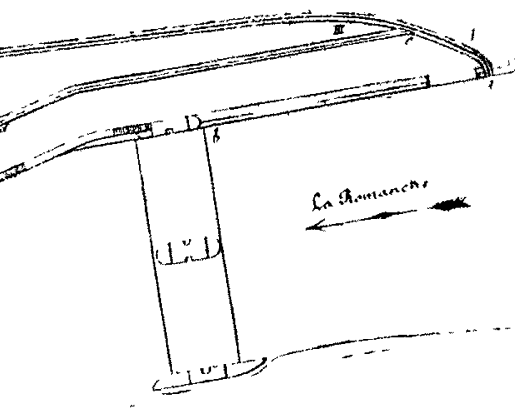

Canal d'amenée. - Le canal d'amenéc fait suite à la chambre VI-VII de la prisc d'eau, et il entre on souterrain presqu'immédiatement aprés la vanne $V$, les pentes ahrutes de la montagne, coupées te nombreux couloirs, n'ayant pas permis de l'établı à ciel ouvert. Sa longueur esi de $2760 \mathrm{~m}$., sa section de $3 \mathrm{~m} 50 \times 3 m 50$, et sa pente de $1,3 \mathrm{Inm}$. par mètre. Il a été percé aulant que possible dans la roche dure, afin d'éviter l'inconvénient de l'étayage pondant la construction, et de la construction d'une voute qui eussent élé nécessaires en terrain meuble. Le tunnel reste parallèle à la vallée, et aussi près que possible de celle-ci; il a élé attaqué à la fois par les deux extrémités, ct par 8 fenêtres intermédiaures.

Les parois du souterrain ont été cimentées pour boucher les fissures et diminuer la perte de charge.

La chamble de mise en charge est toute entière en souterrain. Sa forme générale est sensiblement celle d'un rectangle, de $9 \mathrm{~m} 60$ de largeur et de $30 \mathrm{~m}$. de longueur, perpendiculaire à la vallée. A son raccordement avec le canal 
d'amenée, un déversoir maintient le uiveau de l'eau constant. L'eau qui en provient passe dans une galeric, de $2 \mathrm{~m}$. de largeur, dont l'axe est à $12^{\mathrm{m}} 60$ de colui de la chambre. Cette galerie vient déboucher à l'air libre à mi-hauteur, et forme une cascade yui fait un houreux contraste avec les conduites forcées (voir fig. 10). Elle se continue cusuite par un canal qui traverse la route nationale sous un angle de $70^{\circ}$, el qui vient se jeter dans le canal de fuite.

A son extrémité aval, la chambre de mise cn charge out divisée en deux compartiments, chacun d'eux correspondant à unc conduite forcée. Ces compartimonts ont $3^{m} 60$ de largeur sur $7^{m} \leftleftarrows 0$, de longueur et leur profondeur varie de 3 m50 a $6 \mathrm{~m}$.; ils sont séparés l'un de l'autro par un mur de 1 "'80 d'épaisseur. Une vanne, établic en têle de chaque compartimenl, permet d'isoler chacun d'cux en cas d'avario a 1 une des conduites forcés. lin outre, une grille, a barreaux plus rapprochés que collo de la chambre de decantation, retient les petits corps flottants qui auraient pu passer a travers la première. Une vanno permet d'úvacuer les dernicrs dépots, el au hesoin de vider la chambro en envoyant l'eau dans la şaleric du déversoir.

Conduites forcées. - Les conduites lorcées, de $2 \mathrm{~m} 25$ de diamètre, sont au nombre de deux, ct ont été fournies par la maison Joya, de Grenoble.

Elles sont formées de trois parties rectulignes raccordées par des coudes. La première partic a une longueur

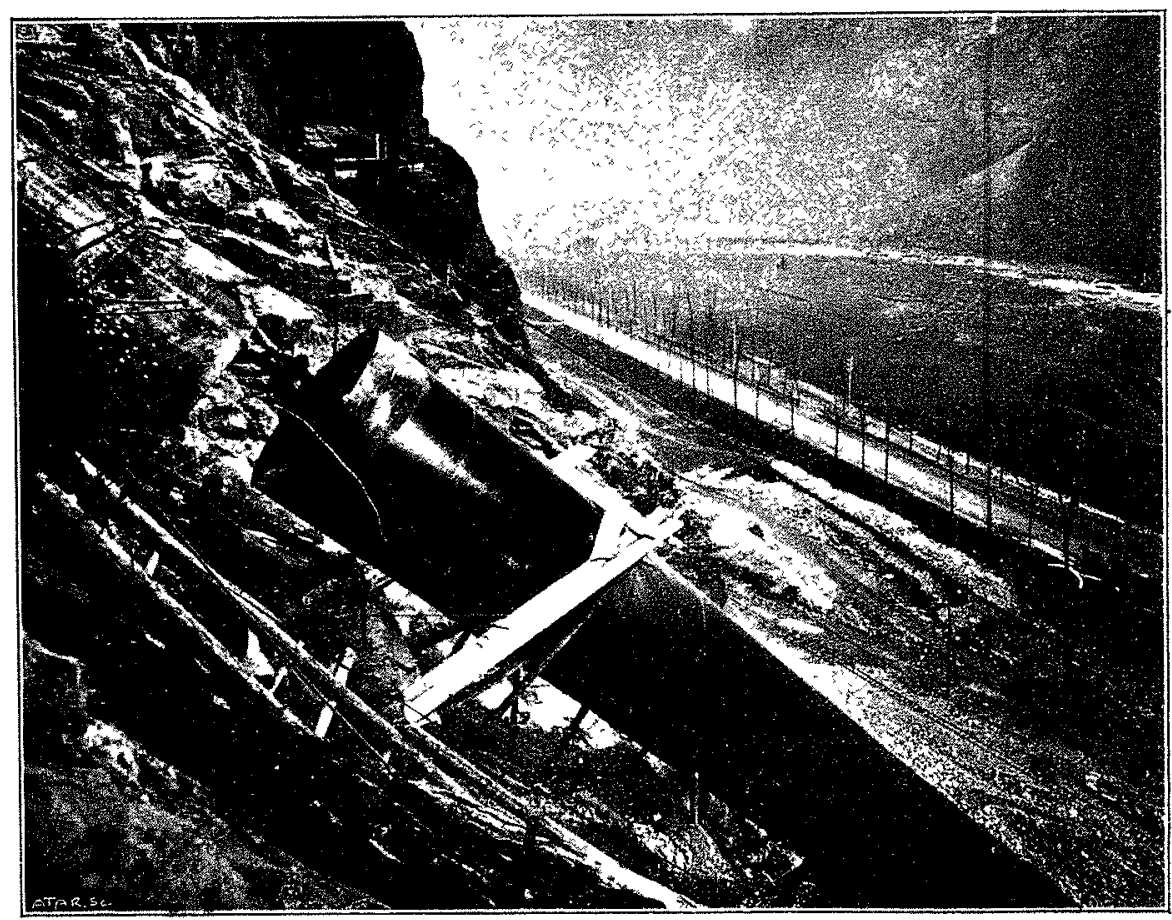

Fig. 5. - Monlage de loune des conduntes furcées.

de $5^{\mathrm{m}} 40$, el solt de la chambre de mise en charge avec une inclinaison de $0 \mathrm{~m} \cdot 40$ par nòtre; son épaisseur est de 6 miljimètres. La deuxième partie, longue de $60 \mathrm{~m} 42$, est inclinée a $45^{\circ}$ cnviron; son épaisseur varie de 6 à $12 \mathrm{~mm}$. linfin, la troisieme partic est horizontale et a une longueur. de $51^{\mathrm{m}} 60$; son épaisseur est de $12 \mathrm{~mm}$. Elle passe dans un acpueduc ménagé sous la route nationale de Grenoble au Bourg-d'Oisans, et vient se raccorder au collecteur perpendiculairement à celui-ci.

Ces condutes sont formées de viroles dacier doux, à double clouire, de $1^{\mathrm{m}} 50$ ) de longueur utile envilon, assemblées les unes aux autros au moyen d'une seule rangée de rivets. Le coude inférieur est constitué par 7 viloles, formant un allgle au centre do $45^{\circ}$, le rayon de ce coude stant de 9 m80. Le coude supérieur est constitué par 5 viroles, formant un angle au contre de $23^{\circ} 46^{\circ}$; le rayon de ce coude est de 12 mètres.
La dislance d'axe en axe des conduites decroit de $5^{\text {in }}$ a au départ de la chambre de mise en charge à 3 m 20 sous h route nationale pour recroill’e jusqu'à 5 "m 20 en arrivant au collecteur.

Les condutes sont supportces par des plliers on macoll. nerie. Pour quelques-uns do cos pilier's, une cornière, livén sur la conduite, prend appui sur cux et empèche tout glissement.

Le collecteur est disposé parallèlement à l'usine. Les conduites forcécs y aboutisscut a angle droil, a sa palla amont. A $3 \mathrm{~m} 7 \mathrm{~s}$ en amont de l'axe de la première conduifo débouche la prive conduisant a la premicre tumbue, ef a $3^{\mathrm{m}} 75$ en aval de la seconde condurte débonche la prise de la seconde turbine les pri-es les autres turbines cont

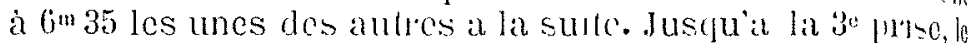
diamètre du collecteur est de 2 m 25 . Lintre la $3^{e}$ et la $4^{\prime \prime}$ prise

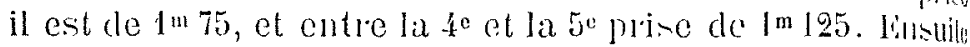
apres, pour les excilallices, il n'chl plus que $0^{\prime \prime} 70$.

Usine génératrice - L'usine gćnératrice eat siluce sul un terreplein remblajé, entre la route latiomale et la Romanche. Elle comprend aclucllement 5 groupes dec. trogènes triphases, de $2000 \mathrm{HL}$, fonctionnant sous ture (lutp de $57^{\mathrm{m}} 50$ a l'etiage et de $55 \mathrm{~m}$. en hautes eaux. L'usine cy prévue pour pouvoir licposer de 8 groupes électrogène, dont 2 de réserve.

L'usine comprend deux corps de batiments : une salle desinachines, de $56 \mathrm{~m}$ de long sur $14 \mathrm{ml}$. de large et $12 \mathrm{~m}$. de hauteur, et un corps anneve. Celui-ci eșt lui-même divisé en deux et com. prend un pavillon d'apparoillage, de $22 \mathrm{~m}$. de long sur $8 \mathrm{ml}$. de lalge, a 3 itages de $4 \mathrm{~m}$. do hauteur chacun, et un atelicr de $17 \mathrm{~m}$. do long sur $14 \mathrm{~m}$ de large, au-dessus duquel se lrouve les bureaux.

Dans le pavillon d'apparcillage, le rez dechaussice, qui se trouve dor ière le lableau di diumbution, est re-ervé à la basse tension; fremer élage contient la laate tension, el fir deuxicme ćlage le dépall des ligrocs. less llansformateurs sont logés dans unc salle a un seul élage, de $22 \mathrm{~m}$. >4 $\mathrm{m}$., siluéc à la sulle do celle róservée à la hasse tencion.

Turbines - Lositubines des alterllateurs on ité fournies par la société des Atelier's piccarl pietet et Cie de Genève. Co sont des lublines Franci-, centripètes parallèles, à axe horizontl lourluant a 375 tours Le réglage est efrectue par une rotation des aubes du distributeur, au nombre de 28, qui sont mobiles autour d'un axe hol'zolt lal. Lorsque le vannage est fermé, toutes los aubes sont inclinées les unes sul les aulres jusqüà se tomcher; au contraire, lorsqu'il est plein, toutes les aules sont palallèles Les diamètres extérjeur et intérieur de la roue molile sont respective. mont de 1 mo el de 0 m80.

Autrefois, les tourillons sur lesculels tommaient les aubes mobiles, et le mecani-me actionnant ces aubs, élaient constainment noyés a l'intérieur de la bàche de li tur]jine. Ils ne pouvaient donc pas être graissés, ef ćlatent, de ce fait, exposés à une destruction plus oll moins rapide. Pour les turluines de l'usine de Séchilienne le mécanisme actionmant les aubes distributrices, visible sur la figure 8, est cnlièrement placé à l'extérieur de la biche de tulbine. Il est hor's de l'eau et visible, et peul ĉtre facılement graissé et réglé pendant la marche de la turljine.

Les aubes directrices sont coulées en acier d'une seule pièce, avec leurs deux tourillons. L'un de ces tourillons pénètre dans une doulle on Jronze, l'autre traverse un presse-étoupe également en bronze ol sort de la turbine. Sur sa partie extérieure, il porte uno manivelle claveléé 
commandée par le servomoteur. La douille et le presseetoupe sont maintenus pleins de graisse consistante au moyen de graisseurs appropriés, de sorte que les tourillons travaillent dans les meilleures conditions.

D'autre part, l'ouverture de chacune des aubes est commandée directement par le mouvement d'une couronne relice au servomoteur au moyen de bielles et manivelles. pur contre, la fermeture de ces aubes est provoquée par la mène couronne, non plus directement, mais par lintermédiaire de ressorts puissants intercalés entre cette coufonne et les manivelles de chacune des aubes $\left(^{*}\right)$. De cette

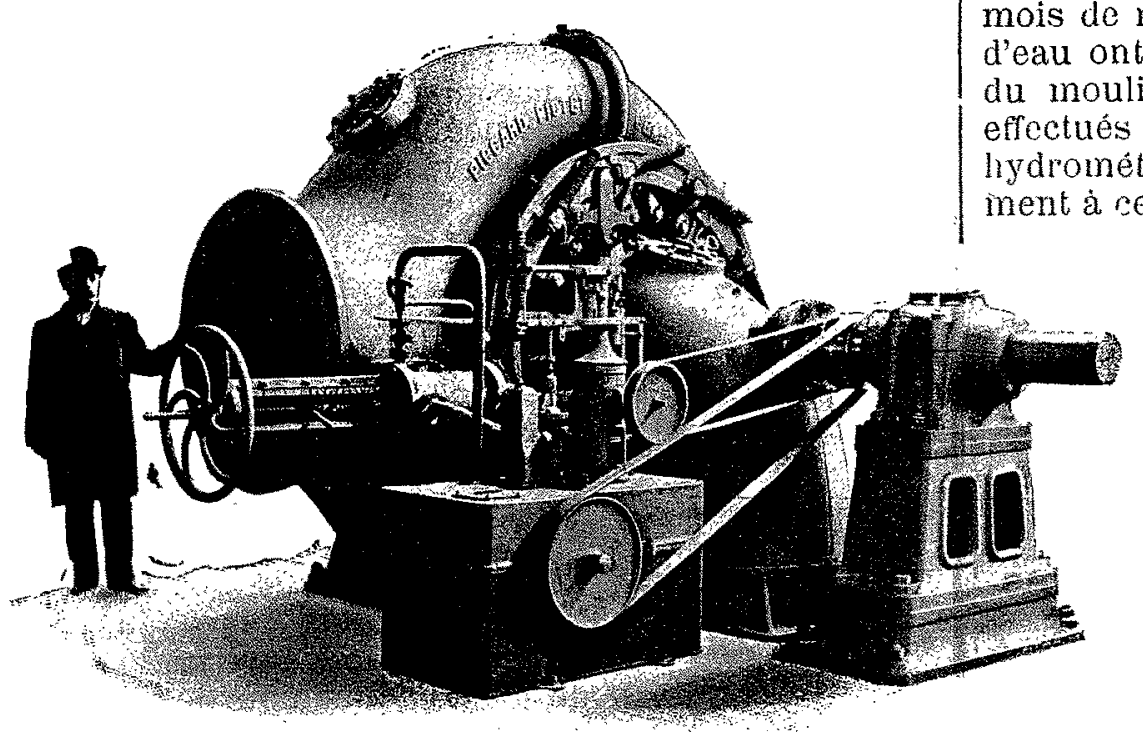

Yu. 6. - Vue dune turbine de $2000 \mathrm{HP}$, aveo son régulateur.

manière, si, pendant la fermeture, un corps étranger vient a. s'introduire entre deux aubes et y reste pincé, les ressorls intéressés cèdent devant cette résistance anormale, et l'aube l'este ouverte au lieu dé se briser. Le mécanisme étant extérieur et visible, l'on peut tout de suite intervenir pour dégager l'aube.

Grace à ce système de commande des aubes mobiles, la surveillance et l'entretien de ces turbines sont grandement facilités, et lusure en est réduite au minimum.

La poussée de la couronne mobile est équilibrée par un

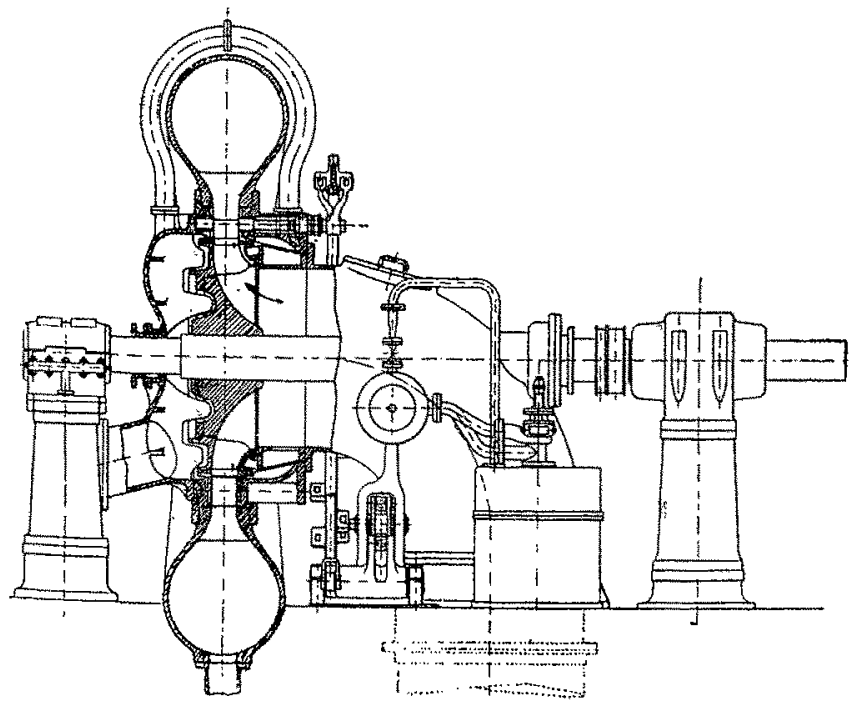

F10. 7. - Coupe suivant laxe d'une turbine.

palier de butée, à huile sous pression, refroidi par une circulation d'eau.

Les rérulateurs sont du système Piccard et Pictet, à servomoteurs à huile sous pression. Chaque régulateur possède

(1) Les ressonts d'iue moitie des aubes sont visibles sur les $0 \mathrm{~g}$. 6 ef 8. Les ressorts de la seconde moilie sont pour la plus grande partic cachís far la conronne. On nappescoil que leur cxlrémité. sa pompe à huile située dans le socle de l'appareil qui sert en mème temps de réservoir d'huile.

Le régulateur proproment dit, ou lachymètre, dont los poids sont suspendus a des ressolts, est d'une cxtréme sensibilité. En fait, l'index de louverture du vannage, visible sur la gauche de la figure 6 , à còté de la manivelle de commande à la main, est en perpétuel mouvement d'oscillations. Le servomoteur est a piston différentiel, et le distributeur est du système à piston fottant commandé par un simple pointeau.

Des essais de rendement très préci ont été exécutês au mois de mai 1907, à l'usine de Séchilienne. Les quantités d'eau ont été mesurées dans le sanal de fuite au mojen du moulinet d'ott perfectionnó. Les jaugeages ont été efrectués sous la direction du Dr Epper, directeur du Burcau hydrométrique fédéral Suisse, qui assistait personnelleinent a ces essais. La charge de l'alternateur était obtenue au moyen d'une résistance liquide avec circulation d'eau, et éfait maintenue constante pendant toute la durée de l'essai.

La maison Piccard, Piclet, avait garanti, pour" les turbines génératrices, les rendements de it et $i 4$ pour 100 a $3 / 4$ et $1 / 2$ charge. Les rendements correspondants ojtenus par ces essais ont été de 81 et 77,5 pour 100.

Les turbines des excitatrices sont centrifuges à libre déviation el peuvent développer $150 \mathrm{HP}$ sous $53^{\mathrm{m}} 50$ do chute utile; olles toument à 500 tours par minute. Le régulateur est à selvomoteur mécanique.

Sous la salle des machines, et jusqu'a do $14 \mathrm{~m}$. cette salle, le canal de fuite est parallèle à l'axe longitudinal de l'usine. Puis il se réunit au canal d'évacuation du déversoir de trop-plein de la chambre d'eau pour retourner à la Romanche.

Chacune des grosses turbines est directement accoupléo au moyen d'un manchon elastique Zoddel à un alternateul. triphasé de 1600 K. V. A. à 4000 volts, 50 périodes, construit

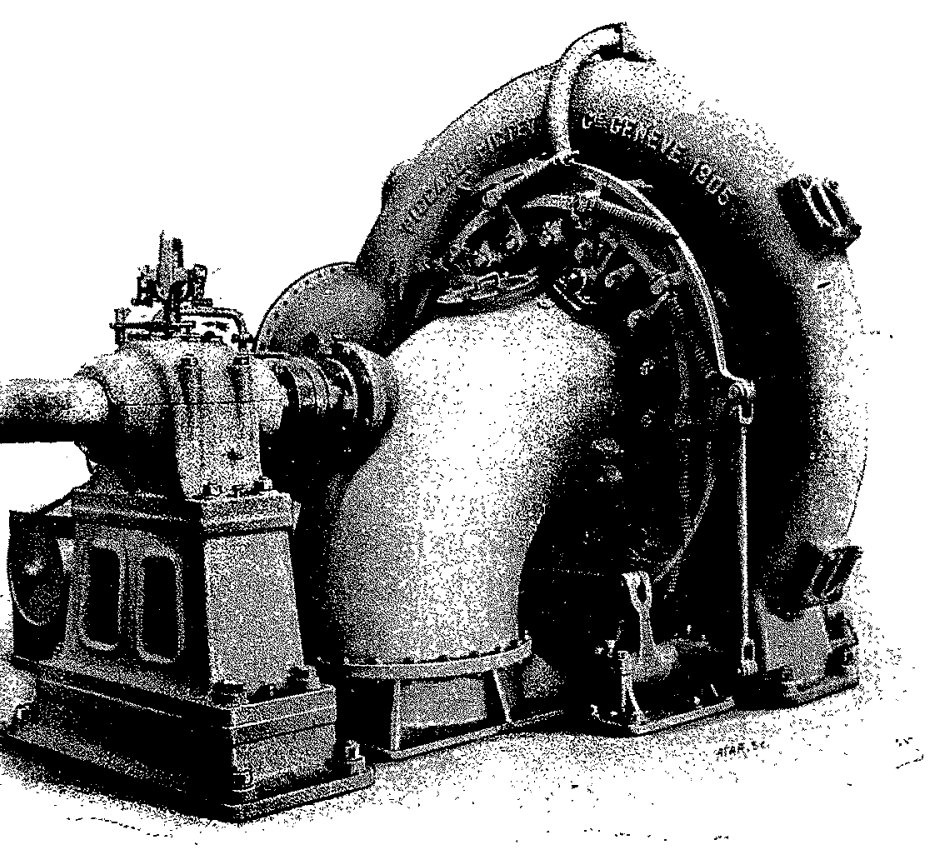

Fig. 8. - Vue du systeme de commande du ramnage

par les Ateliers Schneider of $C^{i e}$, de Champagne-sur-Seine. Un volant, de $3^{\mathrm{m}} 20$ de diamètre, contribue à la régularisation de la vitesse, en cas de surcharge ou décharge brusque des alternateurs.

Alternateurs. - Chaque alternateur est à induit fixe et a inducteur tournant. Il ne comporte pas de bati commun pour ces deux parties, et le réglage de la couronre d'induit 
est independant dẻ celui des paliers supportant l'arbre de l'alternateur. I.e diamètre d'alésagre est de $2 " 20$.

La couronne d'induit est formée de deux parties assemblées, en fonte, portant le circuit magnétique en tôle qui est forlement sorré sur la couromne par des disques rapportés. Des intervalles, destinés à la.ventilation des tôles, sont ménagés daus la masse de ces dernières qui, en outre, portent à leur périphérie intêrieure des alvéoles destinées à recevoir les bobines induites.

La partie inférieure de la couronne d'induit porte deux pattes reposant sur deux caissons formant fondation, et portant des vis de règlage el de rappel qui assurent le centrage de l'induit en hauteur et latéralement par rapport à l'inducteur.

Les bobines induites sont en cuivre de haute conductibilité. Les encoches sont du type demi-frrmé, mais elles ne sont pas symétriques, car les rainures périphériques sont décalćes par rapport à l'axe des encoches, et, dans les 5 paquets de tôle dont est constitué l'induit, ces rainures sont alternces. Ce dispositif diminue les courants de Foucault dans les pièces polaires, qui ont pu ainsi être massives. La courbe de force electromotrice de l'alternateur cst une sinusoïle parfaite.

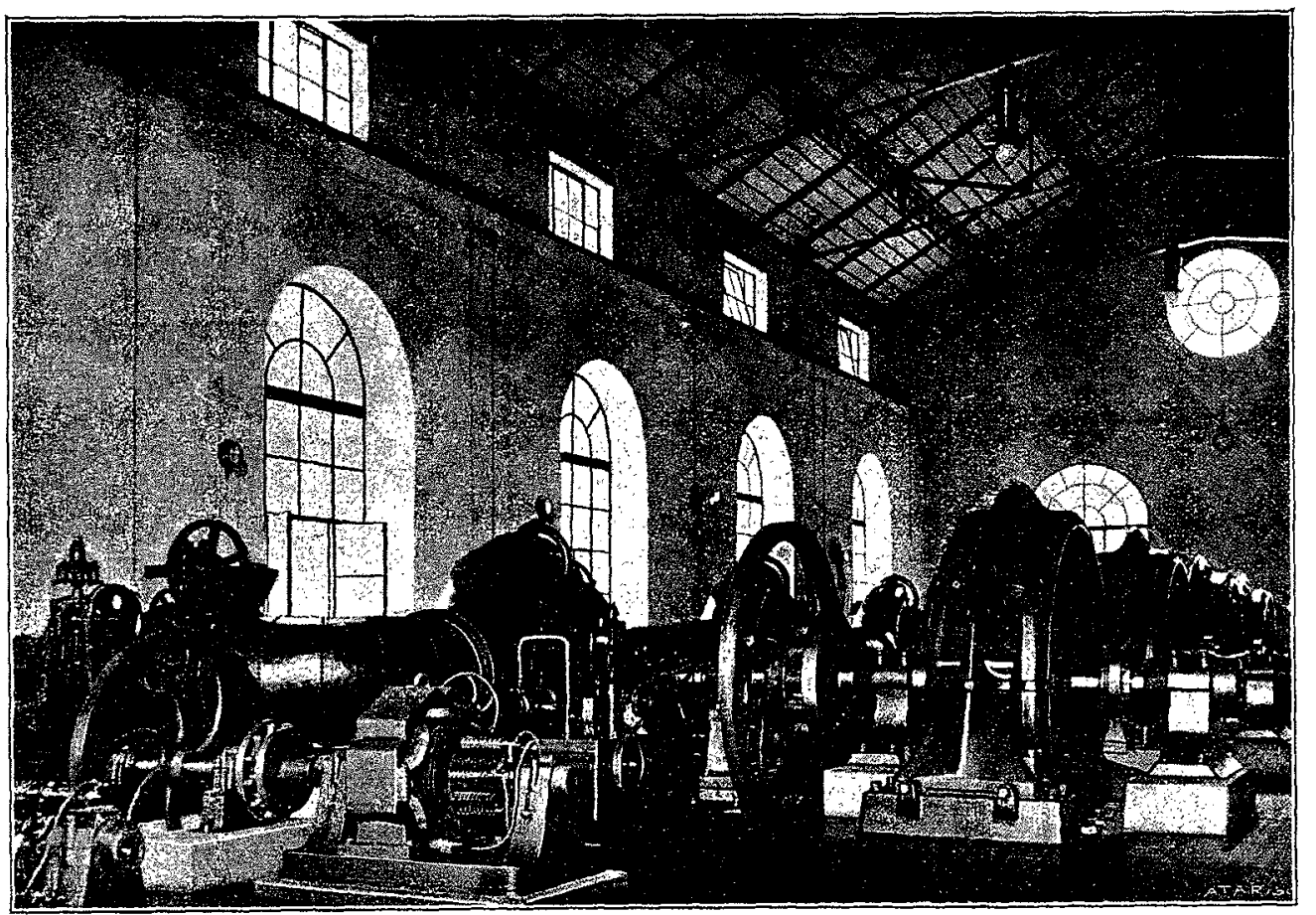

Fıg. 9. - Vue intérieure de la salle des machines.

Les bobines induites traversent le noyau de tôles dans des tubes en micanite qui assurent toute sécurité pour leur isolement à la masse ; la partie extérieure de chaque bobine cst à une distance suffisante des hobines voisines et de la masse pour rendre impossible tout court-cirsuit entre elles el la masse. Les trois phases de l'induit sont connectées en étoile et leurs extrémités aboutissent à 3 bornes montées sur des porcelaines, fix ées à la partie in férieure de la couronne d'induit, et inaccessibles au personnel chargé de la surveillance.

L'inducteur esi constitué par un moyen en acier moulé, claveté sur l'arbre de l'alternateur et portant, venus de fonte, les pôles, de forme oblongue,qui sont au nombre de 16 .

L'enroulement 'inducteur est lormé d'un ruban de cuivre rouge cnroulé sur champ; l'isolement entre spires est obtenu par un tressage du ruban. I.es bolines ainsi constituées sont fortement serrées sur linducteur par les épanouissements polaires, rapportés. et fixés aux póles par deux fortes vis maintenues par des prisonniers. Les extrémités de lenroulement inducteur aboutissent à deux bagues en bronze, calées sur l'arbre de l'allernateur, et recevant le courant d'excitation par des frotleurs en charbon. Le courant d'excitation des alternateurs est fourni par deux dy namos génératrices indépendantes décrites ci-aplès.

L'arbre de lalternateur repose sur deux paliers à stais. sage automatique à bagues et porte, à lune de sesi extrémités, le plateau d"accouplement avec la turbine.

L'élévation maxima de tension entre la marche à pleine charge, avec $\cos \varphi=1$, et la marche à vide, la vitesse res tant constante, est de 8 pour 100.

Ces alternateur's ont été soumis pendant dix minutes à une tension alternative de 8000 volts entre les enroulements jnduits et la masse; ils ont subi une vitesse d'emballement de 630 tours par minute, et ont tourné une heure à 500 tours sans échauffement aux paliers. Le rendement, pour $\cos \varphi=0,85$, a été trouvé aux essais de 94 pour 100 a pleine charge, el de $91,5 \%, 88,5 \%$, et $80 \%$ à $3 / 4,1 / 2$ et $1 / 4$ de charge.

Excitatrices. - L'excitation des alternateurs est lournie par deux dynamos hexapolaires à courant continu, à excitation shunt, de $90 \mathrm{kws}$ chacune, sous 110 volts.

L'inducteur, en acier coulè de grande perméabilité, est en 2 parties seulement, disposition réduisant au minimum le nombre des joints magrnétiques; la partie supérieure est facilement démontable. Les noyaux polaires, venus de fonte avec la carcasse, sont munis de fentes radiales ayant pour effet l'annulation des flux transversaux; sur ces noyaux, sont vissés les épanouissements en fonte arrêtant en position les bobines inductrices. Ces dernières, faites sur mandrins, sont emmanchées sur les pièces polaires.

L'induit est à tambour, de $665 \mathrm{~mm}$. d'alésage, composé d'une couronne circulaire en tôles spéciales, de 3,5 dixièmes de millimètre d'épaissenr', découpées à l'emporte-pièce ; des encoches denil-circulaires sout pratiquées dans ces tôles, et dans les bras du moyeu qui portent la couronne d'induit; des boulons s'ajustent dans ces encoches et assurent l'entraînement.

L'enroulement se compose de barres de cuivre rouge de haute conductibilité, façonnées à l'avance, et interchangeables; ces barres, noyées dans de profondes rainures, sont soustraites à l'action de l'effort tangentiel, évitant ainsi toute chance de détérioration des isolants, et permeltant a l'induit de résister aux emballements. Les parties extérieures des barres forment connexions sans être rabaltues dans les flancs de l'induit, elles assurent ainsi une grande surface de refroidissement et une ventilation énergique.

Transformateurs. - Chaque transformateur débite sur sur le réseau par l'intermédiaire d'un transformateur à bain d'huile et refroidissement par circulation d'eau, qui élève la tension à 26000 volts, l'enroulement primaire étant connecté en éloile et l'enroulement secondaure en triangle. Mais ces transformateurs̀ ont été prévus pour pouvoir suppoltor une tension de $45000 \mathrm{volts}$, les deux enroulements étant connectés en étuile. Leur rendement est de 97,5 pour 100 à pleine charge, et de 97 pour 100 à demi-charge. Ils ont été fournis, ainsi que l'apparcillage, par les Aleliers de Construction Electrique d'uerlikon.

Appareillage. - Le tableau de distribution comprend tous les appareils de mesure et de manouvre usuels, et est situé sur une plateforme dominant la salle des machines. 
Il comprend 14 panneaux de $1 \mathrm{~m}$. de largeur, montés sur une charpente métallique.

Les 5 premiers panneaux en marbre blanc, correspondent chacun à un alternateur et portent un ampèremètre placé sur l'une des phases, un ampèremètre d'excitation, des lampes témoins, le levier de commande d'un interupteur automatique à action difterée, et le volant de manouvro du rhéostat d'excitation. Le panneau suivant comporte les appareils de couplage, lampes de phase, voltmètres, etc. Sur ce panneau se trouvent deux volants qui permettent, soit de manœuvrer toutes ensemble les commandes des rhéostats d'excitation par l'un ou l'autre volant, soil de former deux groupes distincts.

Vieunent ensuite 2 panneaux pour les excitatrices, puis 3 panneaux pour le couplage des transformateurs (les deux premiers correspondent chacun à deux transformateurs). Le panneau qui suit comporte 2 voltmètres donnant la tension des ligues. Les deux derniers panneaux, dont un pour chaque ligne, compoltent trois ampèremètres un waltmètre enregistreur, un compteur, et la commande des interruptcurs de ligne.

Il y a deux sćries de barres omnibus a haute tension permettant d'alimenter séparément, ou en parallèle. les deux lignes avec un transformateur quelconque. Il existe éralement des barres omnibus à basse tension, mais elles ne servent pas qu'accidentellcment, chaque alternateur débitant toujour's en temps normal sur chaque transformateur.

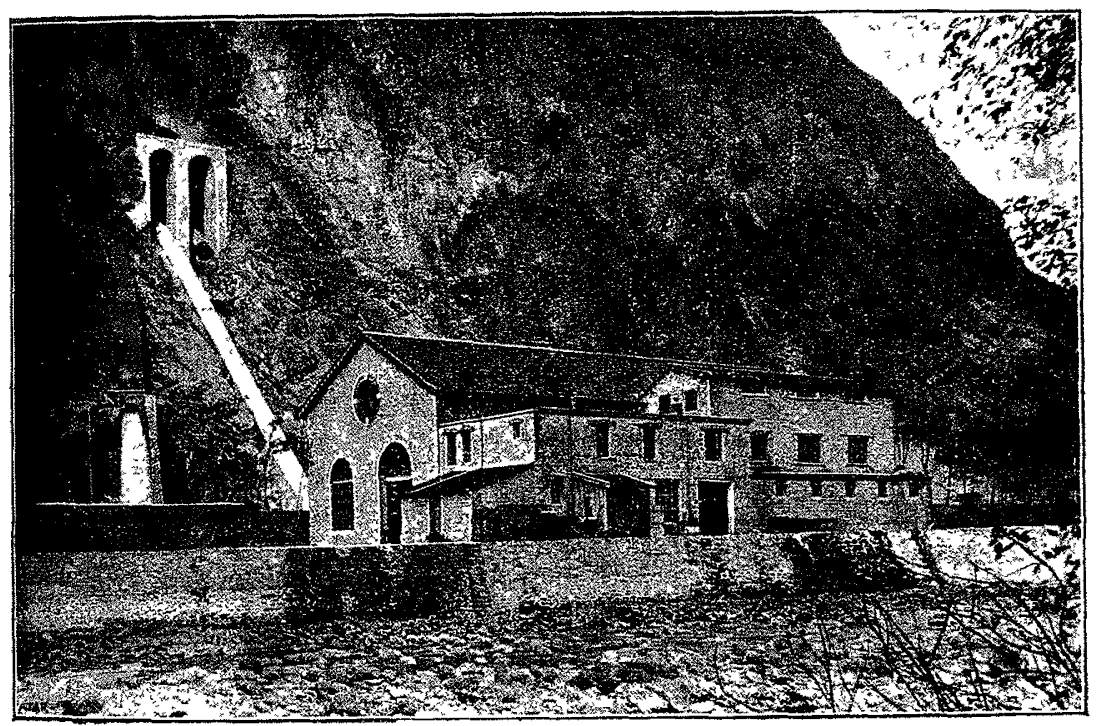

Fla. 10. - Vue générale de l'usine de Síchilienno.

Les interrupteurs de ligne sont établis pour une tension do 45000 volts. 11 s sont à rupture dans l'huile, et sont actionnés par une commande electrique.

Les décliarseurs sont des parafoudres à cornes ordinairos, et sont mis à la terre par l'intermédiaire de résistances liquides. Les limiteurs de teusion sont constitués par des filets d'eau, provenant d'entonnoirs en communication avec chacun des fils de ligne, qui tombent dans une cuve mise à la terre.

Lignes. - Deux lignes, composées chacune de 3 fils de cuivre, de $7 \mathrm{~mm}$ de diamètre, distants de $1 \mathrm{~m}$. les uns des autres, partent de l'usine de séchilienne. Ces deux lignes sont montées sur les mêmes poteaux. Ceux-ci sont métalliques, et ont $10 \mathrm{~m}$. de hauteur au-dessus du sol. Ils sont formés de 4 cornières, sensiblement verticales, assemblées au moyen d'entretoises. Ils ont été construits par la maison Joya. Les isolateurs sont placés sur des traverses en bois formant un cadre à la partie supérieure du poteau.

Ces deux lignes vont se réunir, à Saint-Georges-deCommiers, à colles qui viennent de l'usine d'Avignonnet, pour alimenter le vaste réseau de la Société Grenobloise de Force et Lumière, qui fournit en énergie électrique, non seulement la rogion si industrielle comprise entre Grenoble et Lyon, en passant par Voiron, Bourgoin et Vienne, mais encore-Annonay et ses environs. Nous reviendrons d'ailleur's ultérieurement sur la description de cet important réseau de distribution.

II. Belletet.

\section{LES PHÉNOMÈNES VOLCANIQUES LEURS CAUSES. - LEURS EFFETS}

(SUITE)

\section{$\S$ II. - Causes des manitestations volcaniques.}

Les idées les plus bizarres ont été formulées pour expliquer les phénomènes volcaniques. Aussi, avant d'indiquer quelles sont leurs véritables causes; mentionnons, dans un intérêt à la fois scientifique et historique, quelques-unes des hypothèses qui ont été émises pour les expliquer. Toutes ces hypothèses ont un caractère commun, celui de donner pour point de départ aux phénomènes volcaniques une action chimique dont le premier effet est un dégagement de chaleur. Ainsi que Humboldt l'a fait remarquer, suivant les phases diverses que les sciences chimiques ont parcourues, ces phénomènes_ont été altribués successivement au bitume, aux pyrites, à un mélange humide de fer et de soufre réduits en poussiere.

Les schistes bitumineux, les lignites, certaines houilles, renferment des pyrites qui, une fois mises en contact ayec l'eau superficielie ou atmosphérique, se transforment en sulfate de fer. Cette transformation détermine la production d'une certaine quantité de chaleur, accuséc par la production d'un certain volume de vapcur d'eau qui se dégage des amas de débris accumulés autour des exploitations. Quelquefois les couches de houille ou de lignite s'enflamment spontanément sous l'influence de causes que nous neconnaissons pas d'une manière exacte. Celle inflammation spontanée peut être produite, tantôt par l'oxydation des pyrites placées dans le voisinage dinfiltrations d'eau, tantôt par la combustion du grisou. Quoiqu'il en soit, ces inflammations spontanées allument des incendies souterrains qui durent pendant un temps plus ou moins long, et que Werner appelait des pseudo. volcans.

La mine de Revaux, dans le bassin de Saint-Elienne, peut être citée comre une de celles qui présentent des traces d'ignilion. D'après A. Burat, sous une couche de fer carbonaté, une couche de houille a été en grande partie changée en coke toutes les couches voisines de celles ci paraissent avoir éprouvé aussi une très forte calcination; elles sont rouges et moins dures; le sulfure de fer y a éprouvé une forte sublimation, par suite de laquelle il s'est formé du soufre natif et des veines de sulfate de chaux cristallisé.

Quant à l'hypothèse basée sur le mélange de soufre et de fer, elle repose sur cette expérience de Lémery; tout le monde connait cette réaction chimique dans laquelle on produit une sort de volcan en miniature en mélangeant, dans une cavité creusée dans le sol, du soufre et de la limaille de fer humectée d'eau. Au bout de quelque temps, il se produit un jet comtinu de vapeur d'eau qui s'échappe pendant un temps assez long de l'extrémité supérieure. Mais ca n'est là qu'une expérience de laboratoire, et il y a lieu de s'étonner qu'elle ait pu paraîre suffisante à certains esprits, même il y a 50 ans, pour expliquer le phénomène grandiose d'une éruption volcanique avec émission de laves et projection de scories.

C'est de même que Buffon, frappé de la situation des volcans italiens au voisinage de la mer, pensait que les éruptions du 